\title{
Hybrid Feedback Stabilization of Fuzzy Nonlinear Systems
}

\author{
Yingqi Zhang, ${ }^{1}$ Caixia Liu, ${ }^{1}$ and Xiaowu $\mathrm{Mu}^{2}$ \\ ${ }^{1}$ College of Science, Henan University of Technology, Zhengzhou 450001, China \\ ${ }^{2}$ Department of Mathematics, Zhengzhou University, Zhengzhou 450001, China
}

Correspondence should be addressed to Yingqi Zhang, zyq2018@126.com

Received 24 September 2010; Accepted 8 January 2011

Academic Editor: G. Yin

Copyright () 2011 Yingqi Zhang et al. This is an open access article distributed under the Creative Commons Attribution License, which permits unrestricted use, distribution, and reproduction in any medium, provided the original work is properly cited.

\begin{abstract}
This paper is concerned with the problem of stabilizing one family of fuzzy nonlinear systems by means of fuzzy quantized feedback. The hybrid control strategy originating in earlier work by Brockett and Liberzon (2000) and Liberzon (2003) relies on the possibility of making discrete online adjustments of quantizer parameters. We explore this method here for one class of fuzzy nonlinear systems with fuzzy quantizers affecting the state of the system. New results on the stabilization of the family of fuzzy nonlinear systems are obtained by choosing appropriately quantized strategies. Finally, an illustrative example is given to demonstrate the effectiveness of the proposed method.
\end{abstract}

\section{Introduction}

In recent years, there has been increasing interest in stability analysis and controller design for hybrid and switched systems see, for example, $[1,2]$. In the presence of quantization, the state space of the system is divided into a finite number of quantization regions, each corresponding to a fixed value of the quantizer. At the time of passage from one quantization region to another, the dynamics of the closed-loop system change abruptly. Therefore, systems with quantization can be naturally viewed as hybrid systems. Thus, considerable efforts have been devoted to the study of quantized control, for instance, see [3-7] and the references therein. Among these results, mainly two approaches for studying control problems with quantized feedback are chosen, which are called static quantization policies (e.g., [8-10]) and dynamic quantization policies (e.g., $[5,11])$.

Liberzon [5] gave the conditions of hybrid feedback stabilization of systems with quantized signal under the assumption of the systems being stabilized by a feedback law. De Persis [12] extended Liberzon's [5] results to the problem of stabilizing a nonlinear system by means of quantized output feedback. Gao and Chen [13] presented a new approach to quantized feedback control systems which provided stability and $H^{\infty}$ performance analysis as well as controller synthesis for discrete-time state-feedback control systems with logarithmic quantizers. The most significant feature is the utilization of a quantization dependent Lyapunov function. Ceragioli and De Persis [14] discussed discontinuous stabilization of nonlinear systems with quantized and switching controls, that is, considering the classical problem of stabilizing nonlinear systems in the case of the control laws which take values in a discrete set.

The well-known Takagi-Sugeno (T-S) fuzzy model (e.g., [15]) has been recognized as a popular and powerful tool in approximating and describing complex nonlinear systems. Thus, over the past ten years, the study of T-S systems has been attracting increasing attention, for instance, see [16-23]. However, so far, the study of fuzzy systems with quantized feedback was rare, for instance, [24]. In this paper, we concentrate on the problem of stabilizing fuzzy nonlinear systems via fuzzy quantized feedback. We extend the results (see, [5]) to a class of T-S fuzzy nonlinear systems with general types of quantizers affecting the state of the system. New results on the stabilization of fuzzy nonlinear systems are obtained by choosing appropriately quantized strategies and applying the Lyapunov function approach.

The paper is organized as follows. Section 2 gives the concept of quantizer and the description of fuzzy systems. New results on the stabilization of fuzzy nonlinear systems with fuzzy quantized feedback are presented in Section 3. In Section 4, an example is given to show the effectiveness 
of the proposed method. Conclusions are presented in Section 5.

\section{Problem Statement}

In this section, some notations and definition of quantizer are introduced, and the problem statement is given.

As in [5], a quantizer with general form is defined as follows.

Let $z \in \mathbb{R}^{l}$ be the variable being quantized. A quantizer is defined as a piecewise constant function $q: \mathbb{R}^{l} \rightarrow D$, where $D$ is a finite subset of $\mathbb{R}^{l}$. This leads to a partition of $\mathbb{R}^{l}$ into a finite number of quantization regions of the form $\left\{z \in \mathbb{R}^{l}: q(z)=i\right\}, i \in D$. These quantization regions are not assumed to have any particular shapes. We assume that there exist positive real numbers $M$ and $\Delta$ such that the following conditions hold:

$$
\begin{aligned}
& |z| \leq M \Longrightarrow|q(z)-z| \leq \Delta, \\
& |z|>M \Longrightarrow|q(z)|>M-\Delta .
\end{aligned}
$$

Throughout this paper, we denote by $|\cdot|$ the standard Euclidean norm in the $\mathrm{n}$-dimensional vector space $\mathbb{R}^{n}$ and denote by $\|\cdot\|$ the corresponding induced matrix norm in $\mathbb{R}^{n \times n}$. Condition (1) gives a bound on the quantization error when the quantizer does not saturate. Condition (2) provides a way to detect the possibility of saturation. We will refer to $M$ and $\Delta$ as the range of $q$ and the quantization error, respectively. We also assume that $\{x: q(x)=0\}$ for $x$ in some neighborhood of the origin which is needed to preserve the origin as an equilibrium.

In the control strategy to be developed below, we will use quantized measurements of same the form as in $[3,4]$

$$
q_{\mu}(z):=\mu q\left(\frac{z}{\mu}\right),
$$

where $\mu>0$ is an adjustable parameter, called the "zoom" variable, that is updated at discrete instants of time.

To be convenient, we denoted that $\sum_{i, j=1}^{r}:=\sum_{i=1}^{r} \sum_{j=1}^{r}$, $h_{i}:=h_{i}(x(t)), h_{i}^{q_{\mu}(x)}:=h_{i}\left(q_{\mu}(x(t))\right)$, and $w_{i}:=w_{i}(x(t))$.

The T-S fuzzy system, suggested by Takagi and Sugeno [15] can represent a general class of nonlinear systems. It is based on "fuzzy partition" of input space and it can be viewed as the expansion of piecewise linear partition. Considering a nonlinear dynamic multi-input-multi-output system modeled by the T-S fuzzy system, it can be represented by the following forms.

(i) If-then form:

$R_{i}:$ IF $x_{1}(t)$ is $M_{i 1}, x_{2}(t)$ is $M_{i 2} \ldots$ and $x_{n}(t)$ is $M_{i n}$ then

$$
\dot{x}(t)=A_{i} x(t)+B_{i} u(t) .
$$

$$
\begin{aligned}
\dot{x}(t) & =\frac{\sum_{i=1}^{r} w_{i}\left[A_{i} x(t)+B_{i} u(t)\right]}{\sum_{i=1}^{r} w_{i}} \\
& =\sum_{i=1}^{r} h_{i}(x(t))\left[A_{i} x(t)+B_{i} u(t)\right], \\
w_{i} & =\prod_{j=1}^{n} M_{i j}\left(x_{j}(t)\right), \quad \sum_{i=1}^{r} w_{i}>0, \quad w_{i} \geq 0, \\
h_{i} & =\frac{w_{i}}{\sum_{i=1}^{r} w_{i}}, \quad \sum_{i=1}^{r} h_{i}=1, \quad h_{i} \geq 0,
\end{aligned}
$$

where $x(t)=\left[x_{1}(t), x_{2}(t), \ldots, x_{n}(t)\right]^{T}$ is the state, $u(t) \in \mathbb{R}^{m}$ is the control input, $R_{i}(i=1,2, \ldots, r)$ is the $i$ th fuzzy rule, $r$ is the number of rule, $M_{i 1}, M_{i 2}, \ldots, M_{i n}$ are fuzzy variable, and $h_{i}$ is fuzzy basis function.

For the nonlinear plant represented by (4) or (5), we consider the fuzzy controller as follows.

(iii) If-then form:

$R_{i}:$ if $x_{1}(t)$ is $M_{i 1}, x_{2}(t)$ is $M_{i 2} \ldots$ and $x_{n}(t)$ is $M_{i n}$ then

$$
u(t)=L_{i} x(t)
$$

or

$$
u(t)=L_{i} q_{\mu}(x)
$$

(iv) Input-output form:

$$
u(t) \sum_{i=1}^{r}=h_{i} L_{i} x(t)
$$

or

$$
u(t)=\sum_{i=1}^{r} h_{i}^{q_{\mu}(x)}\left[L_{i} q_{\mu}(x)\right] .
$$

The system (5) with (8) or the system (5) with (9) can, respectively, be written in the form of the T-S fuzzy control system as follows:

$$
\begin{aligned}
\dot{x}(t) & =\sum_{i, j=1}^{r} h_{i} h_{j}\left(A_{i}+B_{i} L_{j}\right) x(t) \\
& =\sum_{i, j=1}^{r} h_{i} h_{j} H_{i j} x(t),
\end{aligned}
$$

or

$$
\begin{aligned}
& \dot{x}(t) \\
& =\sum_{i, j=1}^{r} h_{i} h_{j}^{q_{\mu}(x)}\left\{\left(A_{i}+B_{i} L_{j}\right) x(t)+B_{i} L_{j} \mu\left[q\left(\frac{x}{\mu}\right)-\frac{x}{\mu}\right]\right\} \\
& =\sum_{i, j=1}^{r} h_{i} h_{j}^{q_{\mu}(x)}\left\{H_{i j} x(t)+B_{i} L_{j} \mu\left[q\left(\frac{x}{\mu}\right)-\frac{x}{\mu}\right]\right\},
\end{aligned}
$$

where $H_{i j}$ denotes $H_{i j}:=A_{i}+B_{i} L_{j}$.

(ii) Input-output form: 


\section{Fuzzy Hybrid Feedback Stabilization}

In this section, in order to find some sufficient conditions which stabilize the fuzzy nonlinear systems (11) by choosing appropriately quantized strategies, we require the following assumption 1 and an important lemma is given as in Lemma 1 as follows.

Assumption 1. Assume that there exists a sequence of matrices $\left\{L_{i}\right\}_{i=1}^{r}$ and a common positive definite matrix $P$ and a sequence of positive matrices $\left\{Q_{i j}\right\}_{i, j=1}^{r}$ such that

$$
-Q_{i j}:=\left(A_{i}+B_{i} L_{j}\right)^{T} P+P\left(A_{i}+B_{i} L_{j}\right)=H_{i j}^{T} P+P H_{i j} .
$$

Moreover, both $B_{i}$ and $L_{i}$ for all $i, j \in\{1,2, \ldots, r\}$ are nonzero matrices, which cause no loss of generality because the case of interest is when $A_{i}$ is not a stable matrix for all $i \in\{1,2, \ldots, r\}$.

Remark 1. If Assumption 1 holds, the system (5) with fuzzy control law (8) or the T-S fuzzy system (10) is asymptotically stable by using Lyapunov approach (e.g., see $[16,17])$.

Remark 2. As in [5], it is necessary to suppose that systems are stabilizable. To be convenient, we suppose that Assumption 1 holds so that the system (5) is stabilizable.

Lemma 1. Assume that Assumption 1 holds. an arbitrary $\sigma>$ 0 , and $M$ is large enough compared to $\Delta$ such that

$$
\sqrt{\frac{\lambda_{\min }(P)}{\lambda_{\max }(P)}} M>\Theta_{x} \Delta(1+\sigma)
$$

where

$$
\begin{aligned}
\Theta_{x} & :=2 \theta / \lambda, \\
\lambda & :=\min \left\{\lambda\left(Q_{i j}\right): i, j=1,2, \ldots, r\right\}, \\
\theta & :=\max \left\{\left\|P B_{i} L_{j}\right\|: i, j=1,2, \ldots, r\right\} .
\end{aligned}
$$

Let

$$
\begin{aligned}
& \mathcal{E}_{1}(\mu):=\left\{x: x^{T} P x \leq \lambda_{\min }(P) M^{2} \mu^{2}\right\}, \\
& \mathcal{E}_{2}(\mu):=\left\{x: x^{T} P x \leq \lambda_{\max }(P) \Theta_{x}^{2} \Delta^{2}(1+\sigma)^{2} \mu^{2}\right\} .
\end{aligned}
$$

Then all solutions of (11) that start in the ellipsoid $\mathcal{E}_{1}(\mu)$ enter the smaller ellipsoid $\mathcal{E}_{2}(\mu)$ in finite time.
Proof. We consider the Lyapunov function candidate $V(x)=$ $x^{T} P x$ for the closed-loop system (11) the derivative of $V(x)$ along solutions of (11) is computed as

$$
\begin{aligned}
\dot{V}(x)= & \left(x^{T} P x\right)^{\prime} \\
= & \sum_{i, j=1}^{r} h_{i} h_{j}^{q_{\mu}(x)}\left\{x^{T}\left(H_{i j}^{T} P+P H_{i j}\right) x(t)+2 x^{T} P B_{i} L_{j} \mu\right. \\
& \left.\times\left[q\left(\frac{x}{\mu}\right)-\frac{x}{\mu}\right]\right\} \\
= & \sum_{i, j=1}^{r} h_{i} h_{j}^{q_{\mu}(x)}\left\{-x^{T} Q_{i j} x(t)+2 x^{T} P B_{i} L_{j} \mu\right. \\
& \left.\times\left[q\left(\frac{x}{\mu}\right)-\frac{x}{\mu}\right]\right\} \\
\leq & \sum_{i, j=1}^{r} h_{i} h_{j}^{q_{\mu}(x)}\left\{-\lambda|x|^{2}+2 \theta|x| \mu \Delta\right\} \\
\leq & \sum_{i, j=1}^{r} h_{i} h_{j}^{q_{\mu}(x)}\left\{-\lambda|x|\left(|x|-\Theta_{x} \mu \Delta\right)\right\} \\
= & -\lambda|x|\left(|x|-\Theta_{x} \mu \Delta\right) .
\end{aligned}
$$

According to (13), for any nonzero $x$, we can find a positive scalar $\mu$ such that

$$
\Theta_{x} \mu \Delta(1+\sigma) \leq|x| \leq M \mu .
$$

This is also true in the case of $x=0$, where we set $\mu=0$ as an extreme case and consider the output of the quantizer as zero.

When $\Theta_{x} \mu \Delta(1+\sigma) \leq|x| \leq M \mu$ holds, we have

$$
\left(x^{T} P x\right)^{\prime} \leq-|x| \lambda \Theta_{x} \mu \Delta \sigma .
$$

Claim 1. Both $\varepsilon_{1}(\mu)$ and $\varepsilon_{2}(\mu)$ are invariant sets of the system (11).

Proof of Claim 1. we only prove that $\mathscr{E}_{1}(\mu)$ is an invariant set of the system (11). Assuming $x\left(t_{0}\right) \in \mathscr{E}_{1}(\mu)$, we denote

$$
\tau:=\sup \left\{t: x(t) \in \mathcal{E}_{1}(\mu), \forall t \in\left[t_{0}, \tau\right)\right\},
$$

where $x(t)$ is a solution of the system (11) with the initial condition $x\left(t_{0}\right)$. If $\tau<+\infty$, then there exists a positive constant $\tau$ such that

$$
x(\tau)^{T} P x(\tau)=\lambda_{\min }(P) M^{2} \mu^{2} .
$$

By the virtue of condition (13), we have

$$
\begin{aligned}
\lambda_{\max }(P) \Theta_{x}^{2} \Delta^{2}(1+\sigma)^{2} \mu^{2} & <\lambda_{\min }(P) M^{2} \mu^{2} \\
& =x(\tau)^{T} P x(\tau) \\
& \leq \lambda_{\max }(P)|x(\tau)|^{2} .
\end{aligned}
$$


Hence, we obtain

$$
\Theta_{x} \mu \Delta(1+\sigma)<\left.[|x(t)|]\right|_{t=\tau} .
$$

Using (13), we have $\mathcal{E}_{2}(\mu) \in \mathcal{E}_{1}(\mu)$ and

$$
\begin{aligned}
{\left.[\dot{V}(x(t))]\right|_{t=\tau} } & \leq-\left.\lambda\left[|x(t)|\left(|x(t)|-\mu \Delta \Theta_{x}\right)\right]\right|_{t=\tau} \\
& <-\left.\lambda[|x(t)|]\right|_{t=\tau} \Theta_{x} \mu \Delta \sigma \\
& \leq 0 .
\end{aligned}
$$

By the continuity of $V(x(t))$, there exists a positive constant $\epsilon$ such that for all $t \in[0, \epsilon]$ satisfying

$$
\begin{aligned}
\lambda_{\min }(P)|x(t+\tau)|^{2} & \leq x(t+\tau)^{T} P x(t+\tau) \\
& =V(x(t+\tau)) \\
& \leq V(x(\tau))=\lambda_{\min }(P) M^{2} \mu^{2} .
\end{aligned}
$$

Hence, we have $V(x(t+\tau)) \leq \lambda_{\min }(P) M^{2} \mu^{2}$ for all $t \in[0, \epsilon]$ that is to say that $x(t+\tau) \in \mathcal{E}_{1}(\mu)$ holds for all $t \in[0, \epsilon]$ this is a contradiction with the definition $\tau$. Thus, $\tau=+\infty$. we complete the proof of Claim 1.

Fixed an arbitrary $x\left(t_{0}\right) \in \mathcal{E}_{1}(\mu)$, and for all $x$, we can find a positive scalar $\mu$ satisfying (18). Then, integrating (19) from $t_{0}$ to $t_{0}+T$, we have

$$
\begin{aligned}
x^{T}\left(t_{0}\right. & +T) P x\left(t_{0}+T\right)-x^{T}\left(t_{0}\right) P x\left(t_{0}\right) \\
& =\int_{t_{0}}^{t_{0}+T}\left[x(s)^{T} P x(s)\right]^{\prime} d s \\
& \leq-\int_{t_{0}}^{t_{0}+T}\left[|x(s)| \lambda \Theta_{x} \mu \Delta \sigma\right] d s \\
& \leq-\int_{t_{0}}^{t_{0}+T}\left[\Theta_{x} \mu \Delta(1+\sigma) \lambda \Theta_{x} \mu \Delta \sigma\right] d s \\
& \leq-T \Theta_{x}^{2} \mu^{2} \Delta^{2} \sigma(1+\sigma) \lambda .
\end{aligned}
$$

Hence, we obtain

$$
\begin{aligned}
x^{T}\left(t_{0}+T\right) P x\left(t_{0}+T\right) & \leq x^{T}\left(t_{0}\right) P x\left(t_{0}\right)-T \Theta_{x}^{2} \mu^{2} \Delta^{2} \sigma(1+\sigma) \lambda \\
& \leq \lambda_{\min }(P) M^{2} \mu^{2}-T \Theta_{x}^{2} \mu^{2} \Delta^{2} \sigma(1+\sigma) \lambda
\end{aligned}
$$

If we choose

$$
T:=T_{x}=\frac{\lambda_{\min }(P) M^{2}-\lambda_{\max }(P) \Theta_{x}^{2} \Delta^{2}(1+\sigma)^{2}}{\Theta_{x}^{2} \Delta^{2} \sigma(1+\sigma) \lambda},
$$

we have $x\left(t_{0}+\mathrm{T}_{x}\right) \in \mathcal{E}_{2}(\mu)$.

Using Lemma 1 and assuming that the fuzzy control law (9) of the system (5) satisfies

$$
u(t)= \begin{cases}0, & 0 \leq t<t_{0}, \\ \sum_{i=1}^{r} h_{i}^{q_{\mu}(x)}\left[L_{i} q_{\mu}(x)\right], & t \geq t_{0},\end{cases}
$$

We have the following theorem 1.
Theorem 1. Assume that Assumption 1 holds. Assume that $M$ is large enough compared to $\Delta$ such that

$$
\sqrt{\frac{\lambda_{\min }(P)}{\lambda_{\max }(P)}} M>2 \Delta \max \left\{1, \Theta_{x}\right\}
$$

holds, where $\Theta_{x}$ is the same as in Lemma 1. Then there exists a fuzzy quantized feedback control strategy such that the system (5) with fuzzy quantized control law (9) or the closed fuzzy nonlinear system (11) is globally asymptotically stable.

Proof. The "zooming-out" stage. Let $u=0$. In this case, we rewrite the system (11) for

$$
\begin{aligned}
\dot{x}(t) & =\sum_{i, j=1}^{r} h_{i} h_{j}^{q_{\mu}(x)}\left\{H_{i j} x(t)+B_{i} L_{j} \mu\left[q\left(\frac{x}{\mu}\right)-\frac{x}{\mu}\right]\right\} \\
& =\sum_{i=1}^{r} h_{i} A_{i} x(t) .
\end{aligned}
$$

Let

$$
A:=\underset{\substack{A_{i j} \\ i, j \in\{1,2, \ldots, r\}}}{\operatorname{argmax}}\left\|A_{i j}\right\|
$$

Let $\mu_{0}=\mu(0)=1$, and then increase $\mu$ in a piecewise constant fashion, fast enough to dominate the rate of $e^{r A t}$. Then, there is a time $t \geq 0$ such that

$$
\left|\frac{x(t)}{\mu(t)}\right| \leq \sqrt{\frac{\lambda_{\min }(P)}{\lambda_{\max }(P)}} M-2 \Delta .
$$

By condition (1) in Section 2, it is implied

$$
\left|q\left(\frac{x(t)}{\mu(t)}\right)\right| \leq \sqrt{\frac{\lambda_{\min }(P)}{\lambda_{\max }(P)}} M-\Delta .
$$

We can pick a $t_{0}$ such that (34) holds with $t=t_{0}$. Again, applying conditions (1) and (2) of Section 2, we obtain

$$
\left|\frac{x\left(t_{0}\right)}{\mu\left(t_{0}\right)}\right| \leq \sqrt{\frac{\lambda_{\min }(P)}{\lambda_{\max }(P)}} M .
$$

Hence, we have $x\left(t_{0}\right) \in \varepsilon_{1}\left(\mu\left(t_{0}\right)\right)$ given by (15).

The "zooming-in" stage. Define the sequence of times $\left\{t_{j}\right\}_{j \in \mathbb{N}}$ satisfying

$$
\begin{gathered}
x\left(t_{0}\right) \in \mathcal{E}_{1}\left(\mu\left(t_{0}\right)\right), \\
t_{j+1}=t_{j}+T_{x},
\end{gathered}
$$

and the sequence of positive real numbers

$$
\begin{gathered}
\mu_{0}=\mu\left(t_{0}\right)=1, \\
\mu_{j}=\mu\left(t_{j}\right)=\Omega \mu\left(t_{j-1}\right)=\Omega^{j} \mu_{0}=\Omega^{j} .
\end{gathered}
$$

where $\Omega$ denotes $\sqrt{\lambda_{\max }(P) / \lambda_{\min }(P)}\left(\Theta_{x} \mu \Delta(1+\sigma) / M\right)$ and $T_{x}$ is the same as in (28). 
Define also the control law

$$
u(t)=\sum_{j=1}^{r} h_{j}^{q_{\mu_{j}}(x)} L_{j} q_{\mu_{j}}(x(t)), \quad t \in\left[t_{j}, t_{j+1}\right), j \in \mathbb{N} .
$$

By (30) and Lemma 1 , we have $\Omega<1$ and $\varepsilon_{2}\left(\mu\left(t_{j+1}\right)\right)=$ $\mathcal{E}_{1}\left(\mu\left(t_{j}\right)\right)$. Hence, $\mu_{j}=\Omega^{j} \mu_{0}=\Omega^{j} \rightarrow 0$ as $t \rightarrow+\infty$, and the above analysis implies $x(t) \rightarrow 0$ as $t \rightarrow+\infty$.

In order to prove the stability of the equilibrium $x=0$ of system (11), take an arbitrary $\epsilon>0$ and notice that $u(t)=0$ as $0 \leq t \leq t_{0}$ firstly, finding a positive integer $K:=\ln (\epsilon / M) / \Omega+1, t \in\left[(K-1) T_{x}, K T_{x}\right)$, we have

$$
|x(t)| \leq M \mu\left(t_{K-1}\right)=M \Omega^{K-1} \mu\left(t_{0}\right)=M \Omega^{K-1} \leq \epsilon .
$$

This implies $\mathcal{E}_{1}\left(\Omega^{K-1}\right) \in\{x:|x|<\epsilon\}$.

By the virtue of $q(x)$, there exists a positive constant $\epsilon_{0}$ such that $q(x)=0$ holds for all $x \in\left\{x:|x|<\epsilon_{0}\right\}$. With no loss of generality, we assume $\epsilon_{0} \leq \epsilon$. We define

$$
\delta:=\min \left\{\epsilon_{0} e^{-r\left\|A_{j}\right\| T_{x}}: j=1,2, \ldots, K\right\}=\epsilon_{0} e^{-r\|A\| K T_{x}} .
$$

Then for all $|x(0)|<\delta$ and for all $j=1,2, \ldots, K$, we have

$$
\left|\frac{x(t)}{\mu\left(t_{j}\right)}\right| \leq|x(0)| e^{r\left\|A_{j}\right\| T_{x}} \leq|x(0)| e^{r\|A\| K T_{x}} \leq \epsilon_{0},
$$

Hence there exists a positive constant $\delta:=\epsilon_{0} e^{-r\|A\| K T_{x}}$, and the solutions of $\dot{x}=\sum_{i=1}^{r} h_{i} A_{i} x$ with $|x(0)|<\delta$ stay in the intersection of this $\epsilon_{0}$ with the region $\{x: q(x)=q(x / \Omega)=$ $\left.q\left(x / \Omega^{2}\right)=\cdots=q\left(x / \Omega^{K-1}\right)\right\}=0$ for all $t \in\left[0, K T_{x}\right]$. Therefore, these solutions satisfy $|x(t)| \leq \epsilon$ for all $t \geq 0$.

\section{Numerical Example}

In this section, we consider the following nonlinear system:

$$
\begin{gathered}
\dot{x}_{1}=a x_{1}(t)+b x_{2}(t), \\
\dot{x}_{2}=c x_{2}(t)+d h\left(x_{1}(t)\right) x_{2}(t)+u(t),
\end{gathered}
$$

where $a, b, c, d$ are constants, $u(t)$ is control input, and

$$
h\left(x_{1}(t)\right)= \begin{cases}\frac{\sin x_{1}(t)}{x_{1}(t)}, & x_{1}(t) \neq 0 \\ 1, & x_{1}(t)=0 .\end{cases}
$$

It follows that the nonlinear system can be represented by the following T-S fuzzy model.

(i) If-then rule: if $x_{1}(t)$ is $F_{1}$, then $\dot{x}=A_{1} x(t)+B_{1} u(t)$; IF $x_{1}(t)$ is $F_{2}$, then $\dot{x}=A_{2} x(t)+B_{2} u(t)$, where

$$
\begin{array}{cc}
x(t) & =\left[\begin{array}{l}
x_{1}(t) \\
x_{2}(t)
\end{array}\right], \quad A_{1}=\left[\begin{array}{cc}
a & b \\
0 & c+d
\end{array}\right], \\
A_{2} & =\left[\begin{array}{ll}
a & b \\
0 & c
\end{array}\right], \quad B_{1}=B_{2}=\left[\begin{array}{l}
0 \\
1
\end{array}\right] .
\end{array}
$$

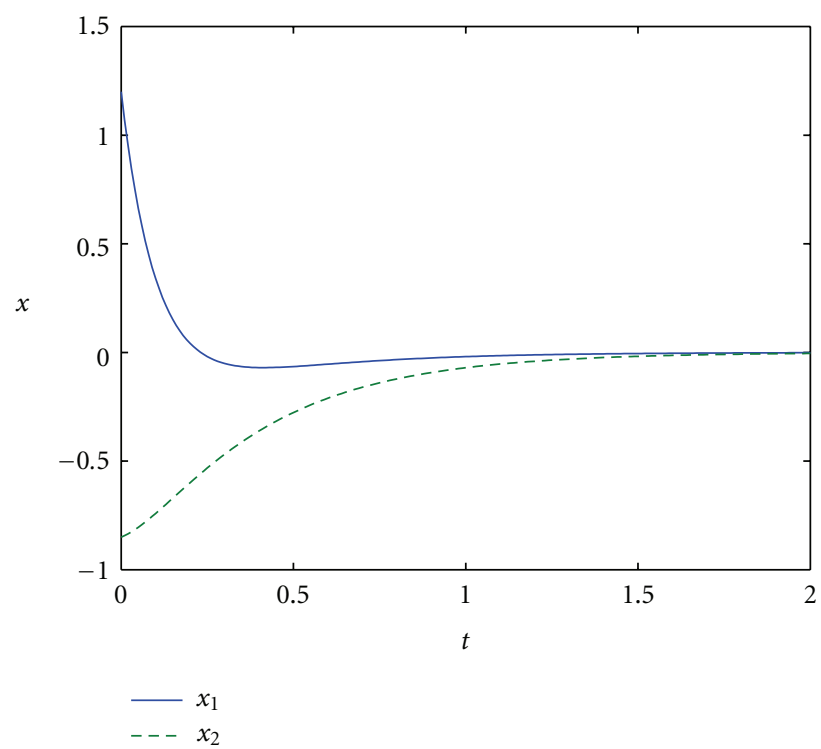

Figure 1: System state.

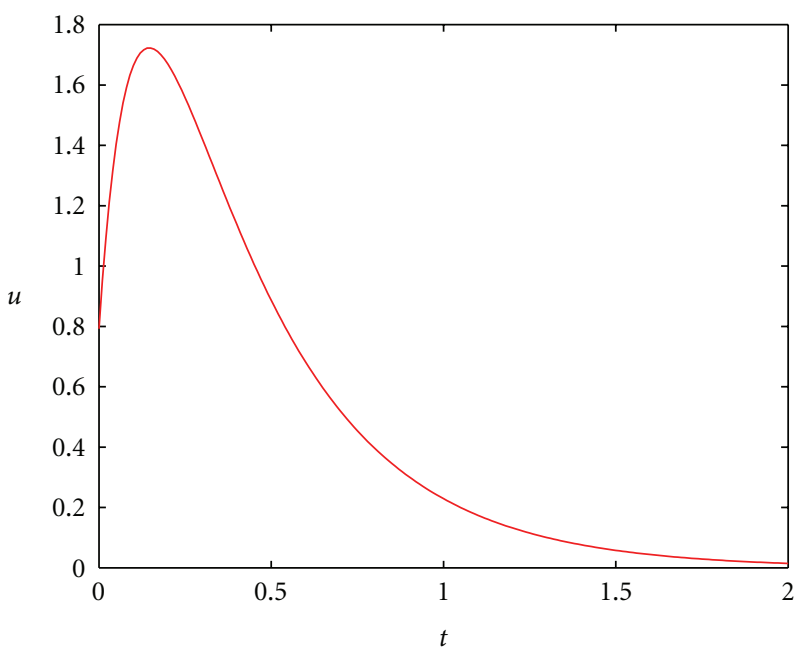

FIGURE 2: System control law.

Moreover, the $F_{1}$ and $F_{2}$ are fuzzy sets defined as $F_{1}(x(t))=$ $h\left(x_{1}(t)\right)$ and $F_{2}(x(t))=1-h\left(x_{1}(t)\right)$.

For the simplicity of simulation, the quantizer is chosen to be logarithmic, which satisfies general quantizer (9), see $[5,9,10]$. That is to say, we choose the quantization level to be described as

$$
U_{\rho}=\left\{ \pm u_{i}, u_{i}=\rho^{i} u_{0}, i=1,2, \ldots\right\} \cup\left\{\rho^{i} u_{0}\right\} \cup\{0\},
$$

and the associated quantizer $q(\cdot)$ is defined as follows:

$$
q(z)= \begin{cases}u_{i}, & \frac{1+\rho}{2} u_{i}<z \leq \frac{1+\rho}{2 \rho} u_{i}, z>0, \\ 0, & z=0, \\ -q(-z), & z<0 .\end{cases}
$$


Thus, the corresponding fuzzy quantized controller can be chosen as

$$
\widehat{u}=q(z)=q\left[\sum_{i=1}^{2} h_{i}(x(t)) \widehat{L}_{i} x(t)\right] .
$$

Now define the quantization error by

$$
e(z)=q(z)-z=\Phi z .
$$

Therefore, $\hat{u}(t)$ can be expressed as

$$
\hat{u}=q(z)=(1+\Phi) q\left[\sum_{i=1}^{2} h_{i}(x(t)) \hat{L}_{\mathrm{i}} x(t)\right],
$$

where $\Phi \in[-\delta, \delta]$. Thus the above closed-loop system with quantized control law can be written as follows

$$
\dot{x}(t)=\sum_{i, j=1}^{2} h_{i} h_{j}\left[A_{i}+(1+\Phi) B_{i} \hat{L}_{j}\right] x(t) .
$$

In this paper, the system parameters are $a=-10, b=2$, $c=0.2, d=0.1$, and quantized parameters are $\delta=0.4$. It can be easily seen that both matrices $A_{1}$ and $A_{2}$ are unstable and the corresponding feedback gain matrix and Lyapunov function matrix of the fuzzy system with quantized controller (49) in Lemma 1 are obtained, respectively:

$$
\widehat{L}_{1}=\widehat{L}_{2}=\left[\begin{array}{l}
-0.9865 \\
-2.0583
\end{array}\right]^{T}, \quad P=\left[\begin{array}{ll}
0.0990 & 0.1216 \\
0.1216 & 1.0403
\end{array}\right] \text {. }
$$

Moreover, for the quantized control of system (42), we can obtain $M>43.8764 \Delta$ from Theorem 1. Then the response of state and control law with quantized control law (49) is showed in Figures 1 and 2, respectively, where the initial condition is $x_{0}=[1.2,-0.85]^{T}$.

\section{Conclusions}

In this paper, we extend the results (see, [5]) to a class of T-S fuzzy nonlinear systems and obtain the conditions of stabilizing a fuzzy nonlinear system via fuzzy quantized feedback. We present new results on the stabilization of fuzzy nonlinear systems by choosing appropriately quantized strategies and applying the Lyapunov function approach. An example has been given to illustrate the effectiveness of the proposed method.

\section{Acknowledgments}

The authors are very grateful to all the anonymous reviewers and the editors for their helpful comments and suggestions. This paper was supported by the National Natural Science Foundation of P. R. China under Grant 60874006, Doctoral Foundation of Henan University of Technology under Grant 2009BS048, by the Natural Science Foundation of Henan Province of China under Grant 102300410118, Foundation of Henan Educational Committee under Grant 2011A120003, and Foundation of Henan University of Technology under Grant 09XJC011.

\section{References}

[1] D. Liberzon and A. S. Morse, "Basic problems in stability and design of switched systems," IEEE Control Systems Magazine, vol. 19 , no. 5, pp. 59-70, 1999.

[2] D. Liberzon, Switching in Systems and Control, Birkhäuser, Boston, Mass, USA, 2003.

[3] R. W. Brockett and D. Liberzon, "Quantized feedback stabilization of linear systems," IEEE Transactions on Automatic Control, vol. 45, no. 7, pp. 1279-1289, 2000.

[4] H. Ishii and B. A. Francis, "Stabilizing a linear system by switching control with dwell time," IEEE Transactions on Automatic Control, vol. 47, no. 12, pp. 1962-1973, 2002.

[5] D. Liberzon, "Hybrid feedback stabilization of systems with quantized signals," Automatica, vol. 39, no. 9, pp. 1543-1554, 2003.

[6] H. Ishii and B. A. Francis, "Quadratic stabilization of sampleddata systems with quantization," Automatica, vol. 39, no. 10, pp. 1793-1800, 2003.

[7] J. Liu and N. Elia, "Quantized feedback stabilization of nonlinear affine systems," International Journal of Control, vol. 77, no. 3, pp. 239-249, 2004.

[8] D. F. Delchamps, "Stabilizing a linear system with quantized state feedback," IEEE Transactions on Automatic Control, vol. 35, no. 8, pp. 916-924, 1990.

[9] N. Elia and S. K. Mitter, "Stabilization of linear systems with limited information," IEEE Transactions on Automatic Control, vol. 46, no. 9, pp. 1384-1400, 2001.

[10] M. Fu and L. Xie, "The sector bound approach to quantized feedback control," IEEE Transactions on Automatic Control, vol. 50, no. 11, pp. 1698-1711, 2005.

[11] S. Tatikonda and S. Mitter, "Control under communication constraints," IEEE Transactions on Automatic Control, vol. 49, no. 7, pp. 1056-1068, 2004.

[12] C. De Persis, "On feedback stabilization of nonlinear systems under quantization," in Proceedings of the 44th IEEE Conference on Decision and Control (CDC '05), pp. 7698-7703, December 2005.

[13] H. Gao and T. Chen, "A new approach to quantized feedback control systems," Automatica, vol. 44, no. 2, pp. 534-542, 2008.

[14] F. Ceragioli and C. De Persis, "Discontinuous stabilization of nonlinear systems: quantized and switching controls," Systems and Control Letters, vol. 56, no. 7-8, pp. 461-473, 2007.

[15] T. Takagi and M. Sugeno, "Fuzzy identification of systems and its applications to modeling and control," IEEE Transactions on Systems, Man and Cybernetics, vol. 15, no. 1, pp. 116-132, 1985.

[16] K. Tanaka and M. Sugeno, "Stability analysis and design of fuzzy control systems," Fuzzy Sets and Systems, vol. 45, no. 2, pp. 135-156, 1992.

[17] K. Tanaka, T. Ikeda, and H. O. Wang, "Fuzzy regulators and fuzzy observers: relaxed stability conditions and LMI-based designs," IEEE Transactions on Fuzzy Systems, vol. 6, no. 2, pp. 250-265, 1998.

[18] H. O. Wang, K. Tanaka, and M. F. Griffin, "An approach to fuzzy control of nonlinear systems: stability and design issues," IEEE Transactions on Fuzzy Systems, vol. 4, no. 1, pp. 14-23, 1996.

[19] H. D. Tuan, P. Apkarian, T. Narikiyo, and Y. Yamamoto, "Parameterized linear matrix inequality techniques in fuzzy control system design," IEEE Transactions on Fuzzy Systems, vol. 9, no. 2, pp. 324-332, 2001. 
[20] C. S. Tseng, B. S. Chen, and H. J. Uang, "Fuzzy tracking control design for nonlinear dynamic systems via T-S fuzzy model," IEEE Transactions on Fuzzy Systems, vol. 9, no. 3, pp. 381-392, 2001.

[21] M. C. M. Teixeira, E. Assunção, and R. G. Avellar, "On relaxed LMI-based designs for fuzzy regulators and fuzzy observers," IEEE Transactions on Fuzzy Systems, vol. 11, no. 5, pp. 613623, 2003.

[22] R. J. Wang, "Observer-based fuzzy control of fuzzy time-delay systems with parametric uncertainties," International Journal of Systems Science, vol. 35, no. 12, pp. 671-683, 2004.

[23] C. Lin, Q. G. Wang, T. H. Lee, and Y. He, "Design of observer-based $H^{\infty}$ control for fuzzy time-delay systems," IEEE Transactions on Fuzzy Systems, vol. 16, no. 2, pp. 534543, 2008.

[24] L. Hou, A. N. Michel, and H. Ye, "Some qualitative properties of sampled-data control systems," IEEE Transactions on Automatic Control, vol. 42, no. 12, pp. 1721-1725, 1997. 

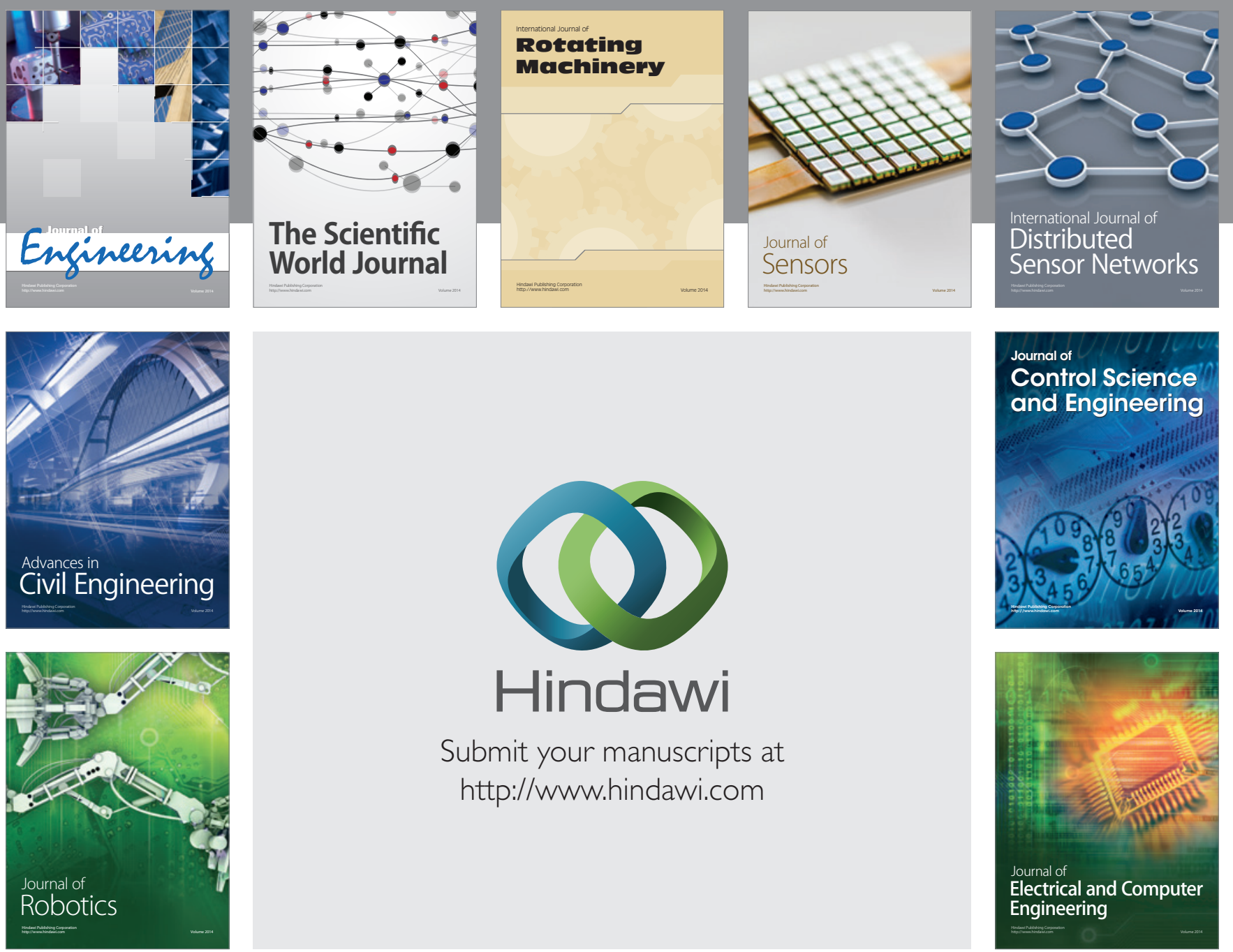

Submit your manuscripts at

http://www.hindawi.com
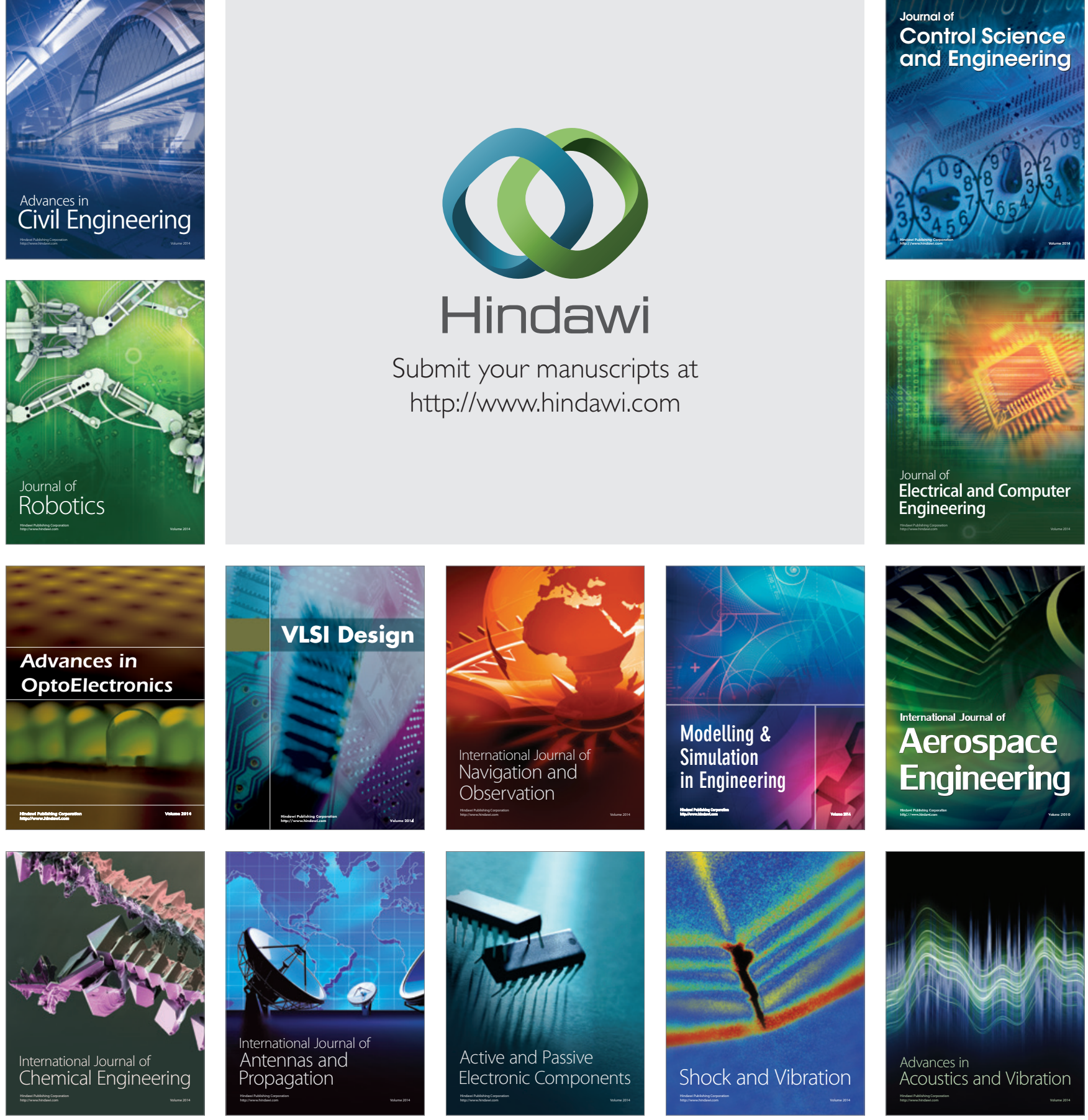